\title{
Insights into the Immunopathophysiology of Severe COVID-19 in Metabolic Disorders
}

\author{
Skand Shekhar ${ }^{1,2} \quad$ Caroline E. Copacino 3 \\ ${ }^{1}$ Section on Endocrinology \& Genetics, Eunice Kennedy Shriver \\ National Institute of Child Health and Human Development, \\ National Institutes of Health, Bethesda, Maryland, United States \\ ${ }^{2}$ Clinical Research Branch, National Institute of Environmental \\ Health Sciences, National Institutes of Health, Research Triangle \\ Park, North Carolina, United States \\ ${ }^{3}$ Tufts University School of Medicine, Boston, Massachusetts, \\ United States \\ 4Endocrinology Division, Internal Medicine Department, University \\ Hospital “Dr. Jose E. Gonzalez," Universidad Autonoma de Nuevo \\ Leon, Monterrey, Mexico \\ 5Plataforma INVEST-KER Unit Mayo Clinic (KER Unit Mexico), School \\ of Medicine, Universidad Autonoma de Nuevo Leon, Monterrey, \\ Mexico \\ ${ }^{6}$ Knowledge and Evaluation Research, Mayo Clinic, Rochester, \\ Minnesota, United States
}

\begin{abstract}
Address for correspondence Skand Shekhar, MD, Section on Endocrinology \& Genetics, Eunice Kennedy Shriver National Institute of Child Health and Human Development and National Institute of Environmental Health Sciences, National Institutes of Health, 10 Center Drive, Room 2N-119, Bethesda, MD 20814, United States (e-mail: skand.shekhar@nih.gov).
\end{abstract}

\author{
Abstract \\ Keywords \\ - COVID-19 \\ - diabetes mellitus \\ - hypertension \\ - obesity
}

Coronavirus disease 2019 (COVID-19) has affected millions of people across the world but disproportionately and severely affects persons with metabolic disorders such as obesity, diabetes mellitus, and hypertension. In this brief review, we discuss the pathways of immune dysregulation that may lead to severe COVID-19 in persons with metabolic conditions.

\section{Introduction}

Coronavirus disease 2019 (COVID-19) is an unprecedented global pandemic caused by the novel severe acute respiratory syndrome coronavirus-2 (SARS-CoV-2) that has affected over 5 million persons globally at the time of this review. ${ }^{1}$ Strikingly, it disproportionately affects persons with underlying endocrine conditions such as obesity, diabetes mellitus, and hypertension. In the United States, the most common co-existing condition affecting patients with COVID-19 is hypertension (49.7\%) followed by obesity (48.3\%) and diabetes mellitus (28.3\%). ${ }^{2}$ These conditions are also associated with an increased risk of poor outcomes including mortality. ${ }^{3-5}$ In light of these risks, it is prudent to explore the pathophysiological mechanisms that account for the observed trends. In this brief review, we discuss the molecular basis of COVID-19 risks in obesity, diabetes mellitus, and hypertension with a focus on immune dysregulation.

\section{Common Immune Dysregulation Pathways}

The human body has multiple protective mechanisms against viral infections. Broadly, viruses gain cellular entry by attaching to viral cell surface receptors. Following this, viral antigens are presented via the major histocompatibility complex to trigger counteractive cellular and humoral immune responses. If immunological mediators are expressed abnormally due to comorbid conditions, the resulting immunological response can be catastrophic. The abnormal expression of immunological mediators appears to be the predominant mechanism in dysregulated immune responses to COVID-19 in metabolic disease that may result in a cytokine storm ( - Fig. 1).

SARS-CoV-2, a betacoronavirus and the causative agent of COVID-19, gains cellular entry by binding to the cell membrane bound angiotensin-converting enzyme 2 (ACE2). ${ }^{6}$ SARS-CoV-2 related coronaviruses such as Middle 


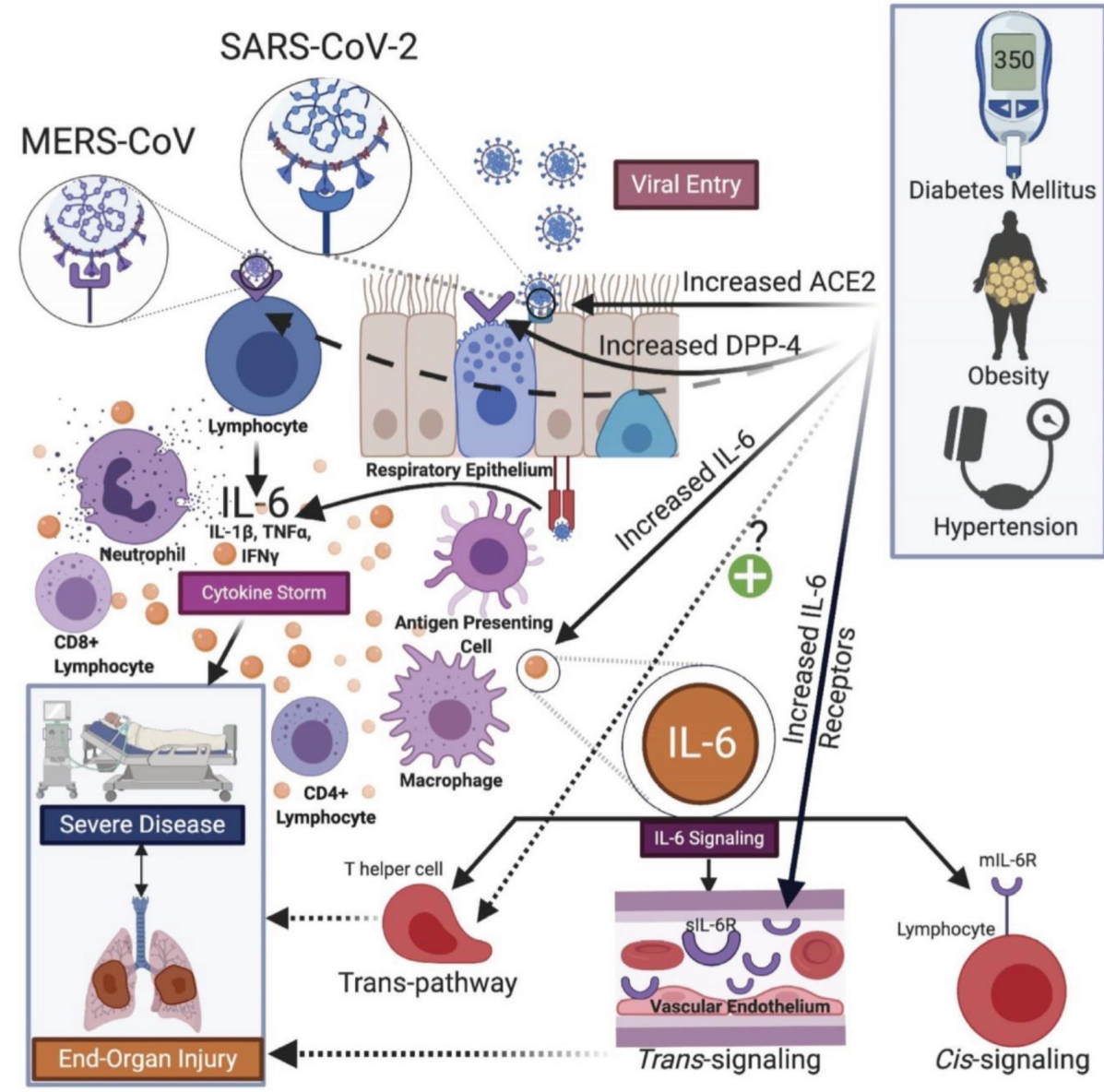

Fig. 1 Immune dysregulation in metabolic disease leading to a pathological immunological coronavirus disease 2019 response. Upstream secretion of interleukin-6 (IL-6) leads to downstream activation of the trans-signaling and trans-pathway modes of IL- 6 action resulting in severe end-organ injury. ACE2, angiotensin-converting enzyme 2; DPP-4, dipeptidyl peptidase-4; MERS-CoV, Middle East respiratory syndrome-coronavirus; mIL-6R, membrane bound interleukin-6 receptor; SARS-CoV-2, severe acute respiratory syndrome coronavirus-2; sIL-6R, soluble interleukin-6 receptor; TNF- $\alpha$, tumor necrosis factor-alpha (image created using BioRenderTM).

East respiratory syndrome-coronavirus (MERS-CoV) and SARS-CoV also directly affect immune cells including monocytes, dendritic cells, and T-cells. ${ }^{7}$ The cellular entry of these betacoronaviruses is mediated by a glucose homeostasis intermediary-dipeptidyl peptidase-4 (DPP-4)-an enzymatic cleaver of glucagonlike peptide- 1 (GLP- 1 ). ${ }^{8}$ Following SARS-CoV-2 infection, immune cell releases interleukin-6 (IL-6), a proinflammatory cytokine. ${ }^{9}$ IL-6 has two main pleiotropic signaling pathways-cis- and trans-signaling. In cis-signaling, IL-6 initially binds to its membrane bound receptor (mIL-6R), found predominantly on the surface of immune cells, followed by recruitment and activation of T-cells, B-cells, and natural killer cells. ${ }^{7}$ IL- 6 secretion by recruited cells is enhanced and its exaggerated release is hypothesized to trigger the onset of the cytokine release syndrome. When IL- 6 binds to its soluble receptor (sIL-6R) present on the vascular endothelium, it leads to the activation of the trans-signaling pathway that in turn causes the release of vascular endothelial growth factor (VEGF) and monocyte chemoattractant protein-1 (MCP-1). Vascular endothelial E-cadherin levels decline as a consequence of trans-signaling, which in combination with elevated VEGF and MCP-1, can lead to increased vascular permeability and leakage
(-Fig. 1). Ultimately, these effects result in syndromes such as acute respiratory distress syndrome and hypotensive shock. A third, less dominant, pathway of IL-6 signaling known as trans-pathway (different from trans-signaling) pathologically activates T-helper cells, a prelude to lung injury and shock. ${ }^{7}$

The pathological mediators of virus-triggered immune responses are elevated in metabolic diseases such as obesity, diabetes mellitus, and hypertension. As an example, IL-6 levels are chronically elevated in obesity and progressively increase with increments in body mass index. ${ }^{10,11}$ This is thought to be consequent to the secretion of IL- 6 by adipocytes in response to chronic adipose tissue hypoxia. ${ }^{12}$ C-reactive protein (CRP), a downstream product of IL-6 and a prognosticator of poor COVID-19 outcomes, is also elevated in obesity. ${ }^{11,13}$ Similarly, IL-6 levels are elevated in diabetes mellitus and insulin resistance states. ${ }^{14}$ Further, IL-6 receptors are also upregulated in type 1 diabetes mellitus, predisposing T-cells to be more sensitive to circulating IL-6. ${ }^{15}$ This leads to a consequent elevation in CRP that is also common in diabetes mellitus. ${ }^{16}$ Equally, IL-6 levels are elevated in hypertension that was well illustrated by Luther et al, who demonstrated the role of angiotensin II as a direct stimulator of IL-6 production. ${ }^{17,18}$ Luther et al also 
reported that the stimulatory effect of IL- 6 on angiotensin II was blocked by the use of angiotensin receptor blockers (ARBs) and mineralocorticoid receptor antagonists such as spironolactone. ${ }^{17}$ Another study of diabetic mice infected with the betacoronavirus, MERS-CoV, reported a prolonged course of severe infection. ${ }^{19}$ This was accompanied by a reduction in CD4+ T-cells and a pathological elevation of IL-17 $\alpha$, a proinflammatory cytokine, confirming the presence of immune dysregulation in diabetes mellitus. ${ }^{19}$

DPP-4, a proinflammatory molecule and a coronavirus receptor, is also elevated in obesity, diabetes mellitus, and hypertension. ${ }^{20}$ DPP-4 is directly proportional to measures of visceral adiposity such as intra-abdominal fat and waisthip ratio. ${ }^{21}$ In persons with diabetes mellitus, DPP-4 levels correlate linearly with glycemic control..$^{22}$ DPP-4 has also been identified as a therapeutic target in diabetes, suggesting a critical role for DPP-4 in the pathophysiology of diabetes. ${ }^{23}$ Finally, the trans-pathway of IL- 6 may be overactive in obesity, diabetes mellitus, and hypertension and may represent the final common pathway in the causative pathophysiology of acute end-organ injury. Taken together, the enhanced secretion and activity of these immune mediators in metabolic diseases are key to understanding the risk of severe COVID-19 (see - Fig. 1).

\section{Specific Immune Dysregulation Pathways}

\section{Obesity}

Obesity is also linked to increased levels of interleukins such as IL- 1 and tumor necrosis factor-alpha (TNF- $\alpha$ ) that may exacerbate the IL-6-mediated immune dysregulation. This is worsened by hyperinsulinemia-induced T-cell dysfunction, a consequence of increased body adiposity. Additionally, obesity is associated with an increased propensity for risks including diabetes mellitus, hypertension, obstructive sleep apnea, and obesity hypoventilation syndrome that further enhance the risks of severe disease by both immune and nonimmune-mediated mechanisms. While DPP-4 inhibition as a therapeutic target to reduce COVID-19 severity remains speculative, GLP-1 receptor analogues have been proven to be immunoregulatory and lung protective in animal models. ${ }^{24}$

\section{Diabetes Mellitus}

Immune dysregulation is multifactorial in diabetes mellitus. In addition to the aforementioned-effects, ACE2 levels are augmented in diabetes mellitus that presumably facilitates viral entry into respiratory and other tissues. ${ }^{25} \mathrm{~A}$ disintegrin and metallopeptidase domain 17 (ADAM17), the enzymatic cleaver of ACE2, is lower in mouse models of diabetes mellitus, which may increase the risk of COVID-19 infection. ${ }^{24,25}$ Co-existing complement deficits, impaired antigen presenting cell function, elevated TNF- $\alpha$ and IL-8, and compromised T-cell function all independently contribute to the dysregulated immune milieu of diabetes mellitus..$^{27-29}$ In addition, the co-existence of other risk factors including obesity and hypertension further amplifies the pre-existing immunological dysfunction in diabetes.

\section{Hypertension}

Hypertension has additional features of immune dysregulation, manifesting as elevated IL-17 and diminished T-cell and natural killer cell function. ${ }^{30,31}$ Hypertension is also associated with an overactive sympathetic drive and elevated angiotensin II, both of which contribute to a compromised immune response. ${ }^{32}$ While sympathetic overdrive has indirect pleiotropic effects on immunity, angiotensin II directly stimulates the secretion of IL-6, which orchestrates the pathological host immune response as detailed above. ${ }^{17,33}$ ACE inhibitors (which are inhibitors of ACE1) and ARBs have been speculated to raise COVID-19 risks through a potential increase in ACE2, but emerging clinical evidence refutes this theory. ${ }^{34-37}$

\section{Conclusion}

Patients with COVID-19 infection in the setting of metabolic diseases such as obesity, diabetes mellitus, and hypertension independently have a significantly worse outcome than those without these diseases. As each of these diseases is associated with dysregulation of the immune system leading to cytokine storm and consequent severe COVID-19. Further research is needed to elucidate the specific immune-deregulatory mechanisms that lead to severe COVID-19 in patients with metabolic disorders.

\section{Funding}

This work was funded by the intramural research program of the Eunice Kennedy Shriver National Institute of Child Health and Human Development (NICHD) and the National Institute of Environmental Health Sciences (NIEHS) of the National Institutes of Health (NIH).

\section{Conflict of Interest}

None declared.

\section{References}

1 Organization WH. Coronavirus disease (COVID-2019) situation reports. Available at: https://www.who.int/emergencies/ diseases/novel-coronavirus-2019. Published 2020. Updated April 30, 2020. Accessed May 1, 2020

2 Garg S, Kim L, Whitaker M, et al. Hospitalization rates and characteristics of patients hospitalized with laboratory-Confirmed Coronavirus Disease 2019 - COVID-NET, 14 States, March 1-30, 2020. Morb Mortal Wkly Rep 2020;69(15):458-464

3 Lighter J, Phillips M, Hochman S, et al. Obesity in patients younger than 60 years is a risk factor for Covid-19 hospital admission. Clin Infect Dis 2020; ciaa415

4 Zhou F, Yu T, Du R, et al. Clinical course and risk factors for mortality of adult inpatients with COVID-19 in Wuhan, China: a retrospective cohort study. Lancet 2020;395(10229):1054-1062

5 Shekhar S, Wurth R, Kamilaris CDC, Eisenhofer G, Barrera FJ, Hajdenberg M, et al. Endocrine Conditions and COVID-19. Horm Metab Res. 2020

6 Lan J, Ge J, Yu J, et al. Structure of the SARS-CoV-2 spike receptor-binding domain bound to the ACE2 receptor. Nature 2020;581(7807):215-220

7 Moore JB, June $\mathrm{CH}$. Cytokine release syndrome in severe COVID-19. Science 2020;368(6490) :473-474 
8 Wang N, Shi X, Jiang L, et al. Structure of MERS-CoV spike receptor-binding domain complexed with human receptor DPP4. Cell Res 2013;23(8):986-993

9 Kang S, Tanaka T, Narazaki M, Kishimoto T. Targeting interleukin-6 signaling in clinic. Immunity 2019;50(4):1007-1023

10 Ellulu MS, Patimah I, Khaza'ai H, Rahmat A, Abed Y. Obesity and inflammation: the linking mechanism and the complications. Arch Med Sci 2017;13(4):851-863

11 Choi J, Joseph L, Pilote L. Obesity and C-reactive protein in various populations: a systematic review and meta-analysis. Obes Rev 2013;14(3):232-244

12 Eder K, Baffy N, Falus A, Fulop AK. The major inflammatory mediator interleukin-6 and obesity. Inflamm Res 2009;58(11):727-736

13 Luo X, Zhou W, Yan X, et al. Prognostic value of C-reactive protein in patients with COVID-19. Clin Infect Dis 2020;ciaa641. Doi: 10.1093/cid/ciaa641

14 Pickup JC, Mattock MB, Chusney GD, Burt D. NIDDM as a disease of the innate immune system: association of acute-phase reactants and interleukin-6 with metabolic syndrome $\mathrm{X}$. Diabetologia 1997;40(11):1286-1292

15 Hundhausen C, Roth A, Whalen E, et al. Enhanced T cell responses to IL- 6 in type 1 diabetes are associated with early clinical disease and increased IL-6 receptor expression. Sci Transl Med 2016;8(356):356ra119

16 Pradhan AD, Manson JE, Rifai N, Buring JE, Ridker PM. C-reactive protein, interleukin 6, and risk of developing type 2 diabetes mellitus. JAMA 2001;286(3):327-334

17 Luther JM, Gainer JV, Murphey LJ, et al. Angiotensin II induces interleukin-6 in humans through a mineralocorticoid receptor-dependent mechanism. Hypertension 2006;48(6):1050-1057

18 Chamarthi B, Williams GH, Ricchiuti V, et al. Inflammation and hypertension: the interplay of interleukin-6, dietary sodium, and the renin-angiotensin system in humans. Am J Hypertens 2011;24(10):1143-1148

19 Kulcsar KA, Coleman CM, Beck SE, Frieman MB. Comorbid diabetes results in immune dysregulation and enhanced disease severity following MERS-CoV infection. JCI Insight 2019;4(20):e131774

20 Stengel A, Goebel-Stengel M, Teuffel P, et al. Obese patients have higher circulating protein levels of dipeptidyl peptidase IV. Peptides 2014;61:75-82

21 Anoop S, Misra A, Bhatt SP, Gulati S, Pandey RM, Mahajan H. High circulating plasma dipeptidyl peptidase- 4 levels in nonobese Asian Indians with type 2 diabetes correlate with fasting insulin and LDL-C levels, triceps skinfolds, total intra-abdominal adipose tissue volume and presence of diabetes: a case-control study. BMJ Open Diabetes Res Care 2017;5(1):e000393

22 Ryskjaer J, Deacon CF, Carr RD, et al. Plasma dipeptidyl peptidase-IV activity in patients with type-2 diabetes mellitus correlates positively with HbAlc levels, but is not acutely affected by food intake. Eur J Endocrinol 2006;155(3):485-493

23 Zhang J, Chen Q, Zhong J, Liu C, Zheng B, Gong Q. DPP-4 inhibitors as potential candidates for antihypertensive therapy: improving vascular inflammation and assisting the action of traditional antihypertensive drugs. Front Immunol 2019;10:1050-1050

24 Zhou F, Zhang Y, Chen J, Hu X, Xu Y. Liraglutide attenuates lipopolysaccharide-induced acute lung injury in mice. Eur J Pharmacol 2016;791:735-740

25 Roca-Ho H, Riera M, Palau V, Pascual J, Soler MJ. Characterization of ACE and ACE2 expression within different organs of the NOD mouse. Int J Mol Sci 2017;18(3):E563

26 Salem ESB, Grobe N, Elased KM. Insulin treatment attenuates renal ADAM17 and ACE2 shedding in diabetic Akita mice. Am J Physiol Renal Physiol 2014;306(6):F629-F639

27 Geerlings SE, Hoepelman AIM. Immune dysfunction in patients with diabetes mellitus (DM) FEMS Immunol Med Microbiol 1999;26(3-4):259-265

28 Calvet HM, Yoshikawa TT. Infections in diabetes. Infect Dis Clin North Am 2001;15(2):407-421

29 Daryabor G, Kabelitz D, Kalantar K. An update on immune dysregulation in obesity-related insulin resistance. Scand J Immunol 2019;89(4):e12747

30 Singh MV, Chapleau MW, Harwani SC, Abboud FM. The immune system and hypertension. Immunol Res 2014;59(1-3):243-253

31 Kvakan H, Luft FC, Muller DN. Role of the immune system in hypertensive target organ damage. Trends Cardiovasc Med 2009;19(7):242-246

32 Abboud FM, Harwani SC, Chapleau MW. Autonomic neural regulation of the immune system: implications for hypertension and cardiovascular disease. Hypertension 2012;59(4):755-762

33 Kenney MJ, Ganta CK. Autonomic nervous system and immune system interactions. Compr Physiol 2014;4(3):1177-1200

34 Meng J, Xiao G, Zhang J, et al. Renin-angiotensin system inhibitors improve the clinical outcomes of COVID-19 patients with hypertension. Emerg Microbes Infect 2020;9(1):757-760

35 Zhang P, Zhu L, Cai J, et al. Association of inpatient use of angiotensin converting enzyme inhibitors and angiotensin II receptor blockers with mortality among patients with hypertension hospitalized with COVID-19. Circ Res 2020. Doi: 10.1161/ CIRCRESAHA.120.317134

36 Mancia G, Rea F, Ludergnani M, Apolone G, Corrao G. Reninangiotensin-aldosterone system blockers and the risk of Covid-19. N Engl J Med 2020. Doi: 10.1056/NEJMoa2006923

37 Reynolds HR, Adhikari S, Pulgarin C, et al. Renin-angiotensinaldosterone system inhibitors and risk of Covid-19. N Engl J Med 2020. Doi: 10.1056/NEJMoa2008975 